\title{
Guessing Facets: Polytope Structure and Improved LP Decoder
}

\author{
Alexandros G. Dimakis, Student Member, IEEE, Amin A. Gohari, Student Member, IEEE, and \\ Martin J. Wainwright, Member, IEEE
}

\begin{abstract}
We investigate the structure of the polytope underlying the linear programming (LP) decoder introduced by Feldman, Karger, and Wainwright. We first show that for expander codes, every fractional pseudocodeword always has at least a constant fraction of nonintegral bits. We then prove that for expander codes, the active set of any fractional pseudocodeword is smaller by a constant fraction than that of any codeword. We further exploit these geometrical properties to devise an improved decoding algorithm with the same order of complexity as LP decoding that provably performs better. The method is very simple: it first applies ordinary LP decoding, and when it fails, it proceeds by guessing facets of the polytope, and then resolving the linear program on these facets. While the LP decoder succeeds only if the ML codeword has the highest likelihood over all pseudocodewords, we prove that the proposed algorithm, when applied to suitable expander codes, succeeds unless there exists a certain number of pseudocodewords, all adjacent to the ML codeword on the LP decoding polytope, and with higher likelihood than the ML codeword. We then describe an extended algorithm, still with polynomial complexity, that succeeds as long as there are at most polynomially many pseudocodewords above the ML codeword.
\end{abstract}

Index Terms-Belief propagation, bit guessing, decimation, error-correcting codes, iterative decoding, linear programming (LP), low-density parity-check (LDPC) codes, linear programming (LP) decoding, pseudocodewords.

\section{INTRODUCTION}

$\mathbf{L}$ OW-density parity-check (LDPC) codes are a class of graphical codes, originally introduced by Gallager [1], that come very close to capacity for large blocklengths even when decoded with the suboptimal sum-product algorithm. The standard techniques for analyzing the sum-product algorithm, including density evolution [2] and EXIT charts [3], are asymptotic in nature (see the book [4] for a comprehensive introduction). For many applications that require low-delay or very low error rates, asymptotic methods may not be suitable for explaining or predicting the behavior of the decoding

Manuscript received June 18, 2007; revised April 28, 2008. Current version published July 15,2009 . This work was supported in part by the NSF by Grant DMS-0528488, Grant CAREER CCF-0545862, by a Microsoft Research Fellowship, and by a UC-MICRO grant through Marvell Semiconductor. This work was presented in part at the International Symposium on Information Theory, Seattle, WA, July 2006.

A. G. Dimakis and A. A. Gohari are with the Department of Electrical Engineering and Computer Science, University of California, Berkeley, CA 94720 USA (e-mail: adim@eecs.berkeley.edu; aminzade@eecs.berkeley.edu).

M. J. Wainwright is with the Department of Electrical Engineering and Computer Science, and the Department of Statistics, University of California, Berkeley, CA 94720 USA (e-mail: wainwrig@eecs.berkeley.edu).

Communicated by T. J. Richardson, Associate Editor for Coding Theory.

Color versions of Figures 1 and 2 in this paper are available online at http:// ieeexplore.ieee.org.

Digital Object Identifier 10.1109/TIT.2009.2023735 algorithms for intermediate block lengths. Feldman, Karger, and Wainwright [5] introduced the linear programing (LP) decoding method, which is based on solving a linear-programming relaxation of the integer program corresponding to the maximum likelihood (ML) decoding problem. In practical terms, the performance of LP decoding is roughly comparable to min-sum decoding and somewhat inferior to sum-product decoding. In particular, for LDPC codes with short to medium block lengths, the LP decoder is comparable to sum-product decoding, whereas for longer block lengths, there seems to be some performance loss relative to sum-product.

However, in contrast to message-passing decoding, the LP decoder either concedes failure on a problem, or returns a codeword along with a guarantee that it is the ML codeword, thereby eliminating any undetected decoding errors. On the conceptual level, the correctness of LP decoding reduces to geometric questions about cost vectors and polytope structure, so that the method is well suited to questions of finite-analysis. Indeed, all of the analysis in this paper applies to finite-length codes.

\section{A. Related Work}

Feldman et al. [5], [6] first introduced and studied the basic idea of LP decoding for turbo and low-density parity check codes. There are various connections to message-passing algorithms [7], [8], including links between the reweighted maxproduct algorithm and dual LP relaxations [8], [9], and the standard max-product algorithm and graph covers [10]. For the binary symmetric channels and suitable expander codes, it has been shown that LP decoding can correct a linear fraction of random [11] or adversarial [12] bit-flipping errors. Koetter and Vontobel [13], [10] established bounds on the pseudoweight for the additive white Gaussian noise (AWGN) channel, showing that it grows only sublinearly for regular codes, and hence that the error probability of LP decoding cannot decay exponentially for the AWGN channel. Subsequent work [14] exploited the constant fraction guarantee [12] to show that LP decoding error decays exponentially for Gaussian channels if the likelihoods are suitably truncated. Other researchers have studied efficient algorithms for solving the LP relaxation, including the reweighted max-product algorithm [9], other forms of iterative dual algorithms [15], and adaptive procedures [16]. As with the work described here, a related line of work has studied various improvements to either standard iterative decoding [17], [18] or to LP decoding [19]-[21]. It should be noted that in a broad sense, the facet guessing algorithms introduced in this paper fall within the class of cutting-plane methods for finding integral 
points of LP relaxations [22]. However, in contrast to many cutting plane methods, our algorithm never introduces new vertices in the polytope, as can happen with many cutting plane methods which introduce new constraints. In independent work, Yang et al. [19] explored branch-and-bound techniques, based on exploring the solution space and generating bounds on the optimal cost. This method can also be viewed within the cutting plane framework, but unlike the methods analyzed here, it does not have polynomial complexity in general. Following the connection between iterative methods and linear programming relaxations, facet guessing is the LP analog of bit guessing and decimation-procedures [23]-[25] used in iterative message-passing algorithms.

\section{B. Our Contributions}

The LP decoder operates by solving a linear program over a polytope $\mathcal{P}$ which constitutes a relaxation of the original combinatorial codeword space. The polytope $\mathcal{P}$, referred in the literature as the relaxed polytope or fundamental polytope, has two types of vertices: integral vertices with 0-1 components corresponding to codewords, and fractional vertices that correspond to pseudocodewords. The first contribution of this paper is to characterize several geometric properties of this relaxed polytope for suitable classes of expander codes. For a given (fractional) pseudocodeword, we define the fractional support as the subset of coordinates that are nonintegral. For general codes, there may exist pseudocodewords with very small fractional supports. Our first result is to show that that for suitable classes of expander codes, the fractional support always scales linearly in the blocklength. In conjunction with known results on the AWGN pseudoweight [13], [10], this fact implies that the size of the minimal nonzero entry in these pseudocodewords must be vanishing at a rate faster than inverse blocklength. In addition, we show that the relaxed polytope $\mathcal{P}$ has the property that many more (a constant fraction of the blocklength) facets are adjacent to integral vertices relative to fractional ones.

Motivated by this geometric intuition, we propose an improved LP decoding algorithm that eliminates fractional pseudocodewords by guessing facets of $\mathcal{P}$, and then decodes by re-solving the optimization problem on these facets. We also provide some theoretical performance guarantees on this improved solver: in particular, for suitable expander codes we prove that it always succeeds as long as there are at most some constant number of pseudocodewords with higher likelihood than the ML codeword. Despite the relative conservativeness of this guarantee, our experimental results show significant performance improvements, particularly at high SNR, for small and moderate blocklengths. In addition, we analyze another type of randomized facet-guessing, still with polynomial complexity, and prove that it succeeds as long as there are at most a polynomial number of pseudocodewords, all adjacent to the ML codeword and with higher likelihood.

Although previous work [13], [10] shows that for the AWGN channel, there do exist pseudocodewords with sublinear pseudoweight, it would be interesting to see if their number can be polynomially or exponentially large. In the first case, if the number of such bad pseudocodewords is bounded by a polynomial, our algorithm could yield an error exponent for AWGN while it is known that LP decoding [10] does not.
The remainder of this paper is organized as follows. In Section II, we provide background on low-density parity check codes and linear programming relaxations for decoding. Section III presents some results on the structure of the LP decoding polytope for suitable classes of expander codes. In Sections IV and V, we describe and analyze improved versions of LP decoding that leverage these structural properties. We conclude with a discussion in Section VI.

\section{BACKGROUND}

In this section, we provide basic background on binary linear codes, factor graphs, and decoding based on linear programming.

\section{A. Low-Density Parity-Check (LDPC) Codes and Factor Graphs}

A binary linear code of blocklength $n$ consists of a linear subspace, where arithmetic is performed modulo two, of the set of all binary sequences $x \in\{0,1\}^{n}$. A code of rate $R=1-\frac{m}{n}$ can be specified by a parity check matrix $H \in\{0,1\}^{m \times n}$ : in particular, the code $\mathbb{C}$ consists of all vectors $x \in\{0,1\}^{n}$ that satisfy $H x=0$ in modulo two arithmetic. Of interest in this paper are low-density parity-check (LDPC) codes [1], meaning that the number of ones in each row and column of the parity check matrix remains bounded independently of the blocklength.

A convenient representation of any LDPC code is in terms of its factor graph [26], [27], a bipartite graph consisting of a set of variable nodes $V=\{1, \ldots, n\}$ corresponding to the columns of $H$, and a set of factor nodes $C=\{1, \ldots, m\}$ corresponding to the rows of $H$. The factor graph has an edge $(i, a)$ between bit $i$ and check $a$ if and only if $H_{a i}$, so that check $a$ imposes a constraint on bit $i$.

\section{B. Decoding Via Linear Programming}

The problem of maximum likelihood (ML) decoding is to determine the most likely codeword on the basis of an observation from a noisy channel. For a binary linear code, the ML decoding problem can be formulated as an integer program of the form

$$
\widehat{x}^{\mathrm{ML}}:=\arg \max _{x \in \mathbb{C}} \sum_{i=1}^{n} \gamma_{i} x_{i}
$$

where $\gamma_{i}$ is a channel-dependent quantity. As a concrete illustration, if the all-zeroes codeword were transmitted over the binary symmetric channel with crossover $\epsilon \in\left(0, \frac{1}{2}\right)$, then $\gamma_{i}=-1$ with probability $1-\epsilon$, and $\gamma_{i}=+1$ with probability $\epsilon$. In the absence of additional structure, the ML decoding problem (1) requires an exhaustive search over an exponentially large space, and is known to be computationally difficult [28]. It is straightforward to convert the ML decoding problem into a linear program over the convex hull of all codewords, a polytope known as the codeword polytope [5]. Unfortunately, for a general binary linear code, the number of constraints (or facets) required to characterize the codeword polytope grows in a nonpolynomial manner with blocklength. Even more strongly, the existence of a polynomial-time separation oracle for the codeword polytope of a general linear code is very unlikely since ML decoding for arbitrary linear codes is NP-hard [28]. 
The technique of LP decoding [5] is based on solving a linear program over a relaxation of the codeword polytope that can be characterized with a polynomial number of inequalities. The standard approach to date is based on a polytope $\mathcal{P}$ obtained by enforcing a set of local constraints associated with each bit. This polytope is referred to as a tree-based relaxation, since it is guaranteed to be exact for any tree-structured factor graph [9].

In order to specify this first-order relaxation, for each check $a \in C$ we define a local codeword polytope (LCP): it is given by the convex hull of the bit sequences that satisfy the check, which are known as local codewords. For example, for a check of degree three, the LCP will be the convex hull of the points $\{000,011,101,110\}$ in the three adjacent variables. One way to write down explicit inequalities for an LCP of general degree is the following: for each check $a \in C$, consider the set of bit sequences $z \in\{0,1\}^{n}$ that fail to satisfy check $a$, meaning that $\oplus_{i \in a} z_{i}=1$. We denote this set of forbidden sequences by $\mathbb{F}(a)$. The local codeword polytope $\operatorname{LCP}(a)$ associated with check $a$ consists of all vectors $f \in[0,1]^{n}$ that are at Hamming distance at least one from each $z \in \mathbb{F}(a)$-viz.

$$
\begin{aligned}
\operatorname{LCP}(a):=\{f \in & {[0,1]^{n} \mid \sum_{i \in N(a) \backslash\left\{k, z_{k}=1\right\}} f_{i} } \\
& \left.+\sum_{i \in\left\{k, z_{k}=1\right\}}\left(1-f_{i}\right) \geq 1 \forall z \in \mathbb{F}(a)\right\} .
\end{aligned}
$$

(Note that any element $f_{j}$ with $j$ not in the neighborhood $N(a)$ of check $a$ is completely unconstrained.) For the 3-check example, the forbidden sequences are $\{001,010,100,111\}$, and the first one corresponds to the inequality $f_{1}+f_{2}+\left(1-f_{3}\right) \geq 1$, assuming the three adjacent variables are $f_{1}, f_{2}, f_{3}$.

We refer to set of $|\vec{F}(a)|$ inequality constraints defining the LCP as the forbidden set inequalities, and we refer to the $2 n$ inequalities $0 \leq f_{i} \leq 1$ as the box inequality constraints. Overall, the relaxed polytope $\mathcal{P}$ is defined as the intersection of the LCPs over all checks-namely, $\mathcal{P}:=\cap_{a \in C} \operatorname{LCP}(a)$. Note that for any check $a$ with degree $d_{c}$, the number of local forbidden sequences is $2^{d_{c}-1}$, so that for a check-regular code the total number of forbidden sequences is $2^{d_{c}-1} m$. For low-density parity-check codes, the maximum degree is bounded so that the relaxed polytope can be described by a linear number of inequalities. (For higher degree checks, there are alternative characterizations of the LCPs that are more efficient than the naive one described here; see the papers [5], [29], for details.)

If the LDPC graph has no cycles, the local forbidden sequences would identify all the possible noncodewords, and the relaxation is guaranteed to be exact by a special case of the junction tree theorem [9], [30]. However, for a general factor graph with cycles, there exist vertices with non $\{0,1\}$ coordinates that satisfy all the local constraints individually, and yet are not codewords (nor linear combinations of codewords). Such sequences are called (fractional) pseudocodewords. To simplify the presentation, in this paper, we use the term pseudocodeword for all vertices of the relaxed polytope, including codewords. The term fractional pseudocodewords then designates the vertices of the relaxed polytope that happen to have at least one fractional coordinate.

\section{Structure of the RelaXed Polytope}

In this section, we turn to some theory concerning the structure of the relaxed polytope. In particular, we begin by addressing the question of the minimal number of fractional coordinates in any fractional pseudocodeword, a quantity that we term the fractional support. Although it is possible to construct codes with an arbitrarily small fractional support, ${ }^{1}$ we show that for expander codes, the fractional support has size linear in blocklength. We then address a second structural property of the polytope: namely, the number of constraints that are active at any vertex. By dimensionality arguments, the size of this active set scales as $\Theta(n)$. Moving beyond this basic observation, we establish that there is a dramatic difference between the active sets associated with fractional pseudocodewords and those associated with (integral) codewords. More specifically, for expander codes, the active set of any fractional pseudocodeword is smaller than the active set size of any codeword by at least a constant fraction (in blocklength) of constraints. We leverage these structural results in Section IV to develop a randomized algorithm for improving the performance of the LP-decoder by guessing facets of the relaxed polytope and resolving the optimization problem.

\section{A. Fractional Support of Pseudocodewords}

The result of this section is to show that the fractional support of any pseudocodeword in any LDPC code defined by an expander graph scales linearly in blocklength. We begin by defining the notion of an expander graph:

Definition 1: Given parameters $\alpha, \delta \in(0,1)$, we say that a $\left(d_{c}, d_{v}\right)$-regular bipartite graph is an $(\alpha, \delta)$ expander if, for all subsets $|S| \leq \alpha n$, there holds $|N(S)| \geq \delta d_{v}|S|$.

Expander graphs have been studied extensively in past work on coding [31], [32], [12], [11]. Indeed, it is well-known that randomly constructed regular LDPC graphs are expanders with high probability (see, e.g., [12]).

The fractional support of a pseudocodeword is defined as follows.

Definition 2: The fractional support of a pseudocodeword $x^{\mathrm{pc}}$ is the subset $V_{\text {frac }}\left(x^{\mathrm{pc}}\right) \subseteq V$ of bits indices in which $x^{\mathrm{pc}}$ has fractional coordinates. Similarly, the subset of checks that are adjacent to bits with fractional coordinates of $x^{\mathrm{pc}}$ is denoted by $C_{\text {frac }}\left(x^{\mathrm{pc}}\right)$.

The following result indicates that all fractional pseudocodewords in an expander code have substantial fractional supports:

Proposition 1: Given an $(\alpha, \delta)$-expander code with $\delta>\frac{1}{2}$, any pseudocodeword has fractional support that grows linearly in blocklength

$$
\left|V_{\text {frac }}\left(x^{\mathrm{pc}}\right)\right| \geq \alpha n \text {, and }\left|C_{\text {frac }}\left(x^{\mathrm{pc}}\right)\right| \geq \delta d_{v} \alpha n .
$$

Proof: The proof exploits the following well-known property [31] of expander graphs.

Unique neighbor property: Given an $(\alpha, \delta)$ expander with $\delta>$ $\frac{1}{2}$, any subset $S \subseteq V$ of size at most $\alpha n$ satisfies the unique

${ }^{1}$ For instance, such a code can be constructed as follows: begin with a small code that has a fractional pseudocodeword, and then add to it $n$ copies of a nonfractional bit, by adding degree two checks and bits. 
neighbor property, i.e there exists $y \in C$ such that $|N(y) \cap S|=$ 1. To establish this claim, we proceed via proof by contradiction: suppose that every $y \in N(S)$ has two or more neighbors in $S$. Then the total number of edges arriving at $N(S)$ from $S$ is at least

$$
2|N(S)|>2 \delta d_{v}|S|>d_{v}|S|
$$

But the total number of edges leaving $S$ has to be exactly $d_{v}|S|$, which yields a contradiction.

We now prove the stated proposition. Consider any set $S$ of fractional bits of size $|S| \leq \alpha n$. Using the expansion and the unique neighbor property, the set $N(S)$ must contain at least one check $a$ adjacent to only one bit in $S$. However, we claim that in any pseudocodeword $x^{\mathrm{pc}}$, no check is adjacent to only one fractional variable node. Indeed, suppose that there were to exist a check adjacent to only one fractional bit: then the associated local pseudocodeword is in the local codeword polytope (LCP) for this check and therefore can be written as a linear combination of two or more codewords [33]. But these local codewords would have to differ in only one bit, which is not possible for a parity check.

Therefore, the check $a$ must be adjacent to at least one additional fractional bit (not in $S$ ). We then add this bit to $S$, and repeat the above argument until $|S|>\alpha n$, to conclude that $\left|V_{\text {frac }}\left(x^{\mathrm{pc}}\right)\right|>\alpha$. Finally, the bound on $\left|C_{\text {frac }}\left(x^{\mathrm{pc}}\right)\right|$ follows by applying the expansion property to a subset of fractional bits of size less than or equal to $\alpha n$.

Remark: In fact, a careful examination of the proof reveals that we can make a slightly stronger claim. Given a pseudocodeword with fractional support $S$, consider the graph $G[S]$ induced by the fractional bits, which may have multiple connected components. The proof of Proposition 1 shows that the size of every connected component must grow linearly in the blocklength for suitable expander codes.

\section{B. Sizes of Active Sets}

For any vertex $v$ of a polytope, its active set $A(v)$ is the set of linear inequalities that are satisfied with equality on $v$. Geometrically, this corresponds to the set of facets of the polytope that contain the vertex $v$. For LP decoding, the set of possible vertices includes both codewords and (fractional) pseudocodewords. The key property that we prove in this section is that for expander codes, codewords have active sets which are larger by at least a constant factor than the active sets of fractional pseudocodewords.

Before stating and proving this result, let us introduce the vertex-facet diagram [33] that describes the relation between the polytope vertices and facets. This diagram can be understood as a bipartite graph $B$ with the set of all codewords and pseudocodewords (vertices of $\mathcal{P}$ ) on its left-hand side, and the set of all constraints (facets of $\mathcal{P}$ ) on its right-hand side. Any given (pseudo)codeword $x^{\mathrm{pc}}$ is connected to a given facet $F$ if and only if $x^{\mathrm{pc}} \in F$; see Fig. 1 for an illustration. In this diagram, the active set $\mathrm{A}\left(x^{\mathrm{pc}}\right)$ of a given pseudocodeword $x^{\mathrm{pc}}$ is simply the set of neighbors of the LHS node representing $x^{\mathrm{pc}}$. The main result of this section concerns the degrees of the LHS nodes, or

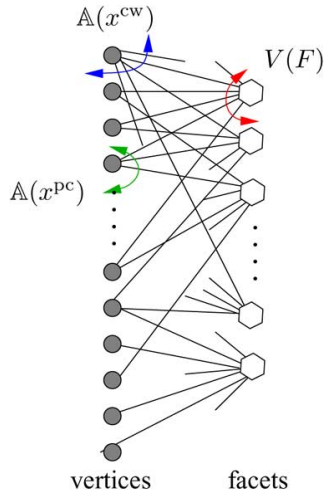

(a)

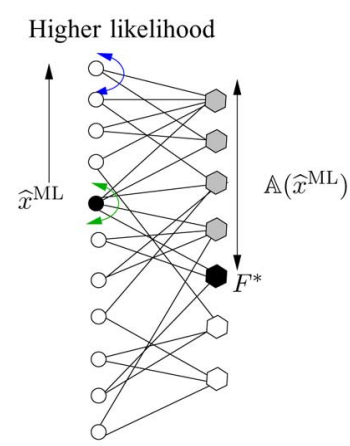

(b)
Fig. 1. (a) Vertex-facet diagram of the relaxed polytope. Circles on the lefthand side (LHS) correspond to vertices (codewords $x^{\mathrm{cw}}$ and fractional pseudocodewords $x^{\mathrm{pc}}$ ) of the relaxed polytope; hexagons on the right-hand side (RHS) correspond to facets (hyperplane inequalities) defining the relaxed polytope. The neighborhood of the LHS node associated with a pseudocodeword $x^{\mathrm{pc}}$ defines its active set $\mathrm{A}\left(x^{\mathrm{pc}}\right)$. (b) Illustration of proof of Corollary 1. Vertices (codewords and pseudocodewords) are ordered by likelihood on LHS. The facet-guessing algorithm fails only if the active set $A\left(\widehat{x}^{\mathrm{ML}}\right)$ of the ML codeword is fully covered by the active sets $\mathrm{A}\left(x^{\mathrm{pc}}\right)$ of pseudocodewords with higher likelihood. Here facet $F^{*}$ remains uncovered so that the algorithm succeeds.

the sizes $\left|A\left(x^{\mathrm{pc}}\right)\right|$ and $\mid A\left(x^{\mathrm{cw}}\right)$ of the (fractional) pseudocodeword and codeword active sets.

Theorem 1: For any $\left(d_{v}, d_{c}\right)$ code with $R \in(0,1)$, the active set of any codeword $x^{\mathrm{cw}}$ has

$$
\left|A\left(x^{\mathrm{cw}}\right)\right|=\gamma_{c w} n
$$

elements. For an $(\alpha, \delta)$-expander code with $\delta>\frac{1}{2}$, the active set of any fractional pseudocodeword $x^{\mathrm{pc}}$ is smaller than the active set of any codeword by a linear fraction-in particular,

$$
\left|\mathrm{A}\left(x^{\mathrm{pc}}\right)\right| \leq n \gamma_{p c}
$$

where the constants are $\gamma_{c w}=\left[(1-R) d_{c}+1\right]$ and $\gamma_{p c}=\left[\left(1-R-\delta d_{v} \alpha\right) d_{c}+2 \delta d_{v} \alpha+(1-\alpha)\right]$. (Note that $\gamma_{p c}<\gamma_{c w}$.)

Proof: We begin by proving (3). By the code-symmetry of the relaxed polytope [5], every codeword has the same number of active inequalities, so it suffices to restrict our attention to the all-zeroes codeword. The check inequalities active at the allzeros codeword are in one-to-one correspondence with those forbidden sequences at Hamming distance 1. Note that there are $d_{c}$ such forbidden sequences, so that the total number of constraints active at the all-zeroes codeword is simply

$$
\left|\mathrm{A}\left(x^{\mathrm{cw}}\right)\right|=m d_{c}+n=n\left[(1-R) d_{c}+1\right],
$$

as claimed.

We now turn to the proof of the bound (4) on the size of the fractional pseudocodeword active set. Recall that the relaxed polytope consists of two types of inequalities: forbidden set constraints (denoted $\mathbb{F}$ ) associated with the checks, and the box inequality constraints $0 \leq x_{i} \leq 1$ (denoted $\mathbb{B}$ ) associated with the bits. The first ingredient in our argument is the fact (see 
Proposition 1) that for an $(\alpha, \delta)$-expander, the fractional support $V_{\text {frac }}\left(x^{\mathrm{pc}}\right)$ is large, so that a constant fraction of the box inequalities will not be active.

Our second requirement is a bound on the number of forbidden set inequalities that can be active at a pseudocodeword. We establish a rough bound for this quantity using the following lemma:

Lemma 1: Suppose that $z$ belongs to a polytope and is not a vertex. Then there always exist at least two vertices $x, y$ such that $\mathbb{A}(z) \subseteq A(x) \cap A(y)$.

PROOF: Since $z$ belongs to the polytope but is not a vertex, it must either belong to the interior, or lie on a face with dimension at least one. If it lies in the interior, then $A(z)=\emptyset$, and the claim follows immediately. Otherwise, $z$ must belong to a face $F$ with $\operatorname{dim}(F) \geq 1$. Then $F$ must contain [33] at least $\operatorname{dim}(F)+1=2$ vertices, say $x$ and $y$. Consequently, since $x, y$ and $z$ all belong to $F$ and $z$ is not a vertex, we must have $A(z) \subseteq A(y)$ and $A(z) \subseteq A(x)$, which yields the claim.

Given a check $c$ and codeword $x^{\mathrm{cw}}$, let $\Pi_{c}\left(x^{\mathrm{cw}}\right)$ denote the restriction of $x^{\mathrm{cw}}$ to bits in the neighborhood of $c$ (i.e., a local codeword for the check $c$ ). With this notation, we have:

Lemma 2: For any two local codewords $\Pi_{c}\left(x^{\mathrm{cw}_{1}}\right)$ and $\Pi_{c}\left(x^{\mathrm{cw}}\right)$ of a check $c$, the following inequality holds

$$
\left|\mathrm{A}\left(\Pi_{c}\left(x^{\mathrm{cw}}\right)\right) \cap \mathrm{A}\left(\Pi_{c}\left(x^{\mathrm{cw}}\right)\right)\right| \leq 2 .
$$

Proof: The intersection $\mathrm{A}\left(\Pi_{c}\left(x^{\mathrm{cw}}{ }_{1}\right)\right) \cap \mathrm{A}\left(\Pi_{c}\left(x^{\mathrm{cw}}{ }_{2}\right)\right)$ is given by the forbidden sequences that have Hamming distance 1 from $\Pi_{c}\left(x^{\mathrm{cw}}{ }_{i}\right), i=1,2$ (i.e., forbidden sequences $f$ such that $d\left(f, \Pi_{c}\left(x^{\mathrm{cw}}{ }_{i}\right)\right)=1$ for $\left.i=1,2\right)$. Thus, if such an $f$ exists, then by the triangle inequality for Hamming distance, we have

$$
\begin{aligned}
2 & \left.=d\left(f, \Pi_{c}\left(x^{\mathrm{cw}}{ }_{1}\right)\right)+d\left(f, \Pi_{c}\left(x^{\mathrm{cw}}{ }_{2}\right)\right)\right) \\
& \geq d\left(\Pi_{c}\left(x^{\mathrm{cw}}{ }_{1}\right), \Pi_{c}\left(x^{\mathrm{cw}}{ }_{2}\right)\right) .
\end{aligned}
$$

But $d\left(\Pi_{c}\left(x^{\mathrm{cw}}\right), \Pi_{c}\left(x^{\mathrm{cw}}\right)\right) \geq 2$ for any two local codewords, so that we must have

$$
d\left(\Pi_{c}\left(x^{\mathrm{cw}_{1}}\right), \Pi_{c}\left(x^{\mathrm{cw}}\right)\right)=2 .
$$

Consequently, we are looking for all the forbidden (odd) sequences of length $d_{c}$ that differ in one bit from two local codewords that are different in two places. Clearly there are only two such forbidden sequences, so that the claim follows.

We can now establish a bound on the size of the active sets of pseudocodewords for $(\alpha, \delta)$-expanders:

Lemma 3: For every pseudocodeword $x^{\mathrm{pc}}$, the size of the active set $\left|A\left(x^{\mathrm{pc}}\right)\right|$ is upper bounded by

$$
\left(m-\left|C_{\text {frac }}\left(x^{\mathrm{pc}}\right)\right|\right) d_{c}+2\left|C_{\text {frac }}\left(x^{\mathrm{pc}}\right)\right|+n-\left|V_{\text {frac }}\left(x^{\mathrm{pc}}\right)\right| .
$$

Proof: The proof is based on the decomposition:

$$
\left|\mathbb{A}\left(x^{\mathrm{pc}}\right)\right|=\left|\mathbb{A}\left(x^{\mathrm{pc}}\right) \cap \mathbb{F}\right|+\left|\mathbb{A}\left(x^{\mathrm{pc}}\right) \cap \mathbb{B}\right| .
$$

The cardinality $\left|\mathbb{A}\left(x^{\mathrm{pc}}\right) \cap \mathbb{B}\right|$ is equal to the number of integral bits in the pseudocodeword, given by $n-\left|V_{\text {frac }}\left(x^{\mathrm{pc}}\right)\right|$. We now turn to upper bounding the cardinality $\left|A\left(x^{\mathrm{pc}}\right) \cap \mathbb{F}\right|$. Consider the $m-\left|C_{\text {frac }}\left(x^{\mathrm{pc}}\right)\right|$ checks that are adjacent to only integral bits of $x^{\mathrm{pc}}$. For each such check, exactly $d_{c}$ forbidden set constraints are active, thereby contributing a total of

$$
d_{c}\left[m-\left|C_{\text {frac }}\left(x^{\mathrm{pc}}\right)\right|\right]
$$

active constraints. Now consider one of the remaining $\left|C_{\text {frac }}\left(x^{\mathrm{pc}}\right)\right|$ fractional checks, say $c$. Consider the restriction $\Pi_{c}\left(x^{\mathrm{pc}}\right)$ of the pseudocodeword $x^{\mathrm{pc}}$ to the check neighborhood of $c$. Since $\Pi_{c}\left(x^{\mathrm{pc}}\right)$ contains fractional elements, it is not a vertex of the local codeword polytope associated with $c$. Therefore, by combining Lemmas 1 and 2, we conclude that

$$
\left|\mathrm{A}\left(\Pi_{c}\left(x^{\mathrm{pc}}\right)\right)\right| \leq 2 .
$$

Overall, we conclude that the upper bound (5) holds.

Using Lemma 3 and Proposition 1, we can now complete the proof of Theorem 1. In particular, we re-write the RHS of the bound (5) as

$$
(1-R) d_{c} n-\left(d_{c}-2\right)\left|C_{\text {frac }}\left(x^{\mathrm{pc}}\right)\right|+n-\left|V_{\text {frac }}\left(x^{\mathrm{pc}}\right)\right| .
$$

From Proposition 1 , we have $\left|C_{\text {frac }}\left(x^{\mathrm{pc}}\right)\right| \geq d_{v} \delta \alpha n$ and $\left|V_{\text {frac }}\left(x^{\mathrm{pc}}\right)\right|>\alpha n$, from which the bound (4) follows.

\section{IMPROVED LP DECODING}

Various improved decoding algorithms have been suggested in past work, both based on extensions of standard iterative decoding (e.g., [17], [18], as well as extensions of LP decoding [21], [19]). Based on the structural results that we have obtained, we now describe an improved decoding algorithm for which some finite-length theoretical guarantees can be made. We begin with some simple observations: (i) ML decoding corresponds to finding the vertex in the relaxed polytope that has the highest likelihood and integral coordinates; and (ii) Standard LP decoding succeeds if and only if the ML codeword has the highest likelihood over all pseudocodewords.

These observations highlight the distinction between LP decoding and ML decoding. An LP solver, given the (polynomially many) facets of the relaxed polytope, determines the vertex with the highest likelihood without having to go through all the exponentially many vertices of $V$. In contrast, the ML decoder can go down this list, and determine the first vertex which has integral coordinates. This motivates facet-guessing: suppose that there exists only one fractional pseudocodeword $x^{\mathrm{pc}}{ }_{1}$ that has higher likelihood than the ML codeword $\widehat{x}^{\mathrm{ML}}$. The LP decoder will output the pseudocodeword $x^{\mathrm{pc}}$, resulting in a decoding error. However, now suppose that there exists a facet $F_{1} \in A$ such that $\widehat{x}^{\mathrm{ML}} \in F_{1}$ but $x^{\mathrm{pc}} \notin F_{1}$. Consider the reduced polytope $\mathcal{P}^{\prime}$ created by restricting the relaxed polytope $\mathcal{P}$ to the facet $F_{1}$ (i.e., $\mathcal{P}^{\prime}=\mathcal{P} \cap F_{1}$ ). This new polytope will have a vertex-facet graph $\mathcal{B}^{\prime}$ with vertices $V^{\prime}=N\left(F_{1}\right)$ i.e., all the vertices that are contained in $F_{1}$. The likelihoods will be the same, but $p_{1}$ will not belong in $\mathcal{P}^{\prime}$ and therefore we can use an LP solver to determine the vertex with the highest likelihood in $\mathcal{P}^{\prime}$. If we had chosen the correct facet, this vertex would be the ML codeword $\widehat{x}^{\mathrm{ML}}$. Based on this intuition, we now formally describe the facet-guessing algorithm for improved LP decoding. 


\section{Facet Guessing Algorithm}

1) Run LP decoding: if outputs an integral codeword, terminate. Otherwise, go to Step 2).

2) Take as input:

- fractional pseudocodeword $x^{\mathrm{pc}}$ from the LP decoder;

- likelihood vector $\gamma$.

3) Given a natural number $N \geq 1$, repeat for $i=1, \ldots N$ :

(a) Select a facet $F_{i} \in\left(A \backslash A\left(x^{\mathrm{pc}}\right)\right.$, form the reduced polytope $\mathcal{P}^{\prime}=\mathcal{P} \cap F_{i}$.

(b) Solve the linear program with objective vector $\gamma$ in $\mathcal{P}^{\prime}$, and save the optimal vertex $z_{i}$.

4) From the list of optimal LP solutions $\left\{z_{1}, \ldots, z_{N}\right\}$, output the integral codeword with highest likelihood.

\section{Remarks:}

(a) We can consider two variations of facet guessing: exhaustive facet guessing $(\mathrm{EFG})$ tries all possible facets (i.e., $\left.N=\left|\left(\mathcal{A} \backslash A\left(x^{\mathrm{pc}}\right)\right)\right|\right)$, while randomized facet guessing $(\mathrm{RFG})$ randomly samples from $\left(A \backslash A\left(x^{\mathrm{pc}}\right)\right)$ a constant number of times (e.g., $N=20$ ).

(b) Regardless of the problems, the exhaustive facet-guessing (EFG) algorithm has polynomial-time complexity, since the number of calls to the LP solver grows linearly as

$$
\left|\mathrm{A} \backslash \mathrm{A}\left(x^{\mathrm{pc}}\right)\right|=\mathcal{O}(n) .
$$

On the other hand, the RFG algorithm requires only a constant number of calls to an LP solver, and therefore has the same order of polynomial complexity as standard LP decoding. When these algorithms are applied to a sequence of decoding problems, one would expect that the average complexity is typically very close to LP decoding, since the facet-guessing routines (Step 2) run only if the LP decoder has already failed.

We now provide a simple necessary and sufficient characterization for the EFG algorithm to fail.

Lemma 4: The exhaustive facet-guessing algorithm fails to find the ML codeword $\widehat{x}^{\mathrm{ML}} \Longleftrightarrow$ every facet $F \in A\left(\widehat{x}^{\mathrm{ML}}\right)$ contains a fractional pseudocodeword with likelihood greater than $\widehat{x}^{\mathrm{ML}}$.

Proof: Denote the set of fractional pseudocodewords with likelihood higher than $\widehat{x}^{\mathrm{ML}}$ by $\mathbb{P}\left(\widehat{x}^{\mathrm{ML}}\right)$. Assume there exists a facet $F_{i}$ such that $\widehat{x}^{\mathrm{ML}} \in F_{i}$ and $x^{\mathrm{pc}} \notin F_{i}$ for all pseudocodewords $x^{\mathrm{pc}} \in \mathbb{P}\left(\widehat{x}^{\mathrm{ML}}\right)$. Then the facet-guessing algorithm will at some round select the facet $F_{i}$, and the LP solver will output $\widehat{x}^{\mathrm{ML}}$, as the vertex in $\mathcal{P}^{\prime}$ with the highest likelihood. Consequently, the ML solution $\widehat{x}^{\mathrm{ML}}$ will be in the list of LP solutions in step (4). Since $\widehat{x}^{\mathrm{ML}}$ is the ML codeword, there can be no other integral codeword with higher likelihood in the list, so that the algorithm must output $\widehat{x}^{\mathrm{ML}}$. Conversely, suppose that every facet $F \in A\left(\widehat{x}^{\mathrm{ML}}\right)$ contains a fractional pseudocodeword with likelihood greater than $\widehat{x}^{\mathrm{ML}}$. Then, the ML codeword $\widehat{x}^{\mathrm{ML}}$ will never be the output of the LP solver at any round, since some pseudocodeword will always have higher likelihood. Consequently, the ML codeword will not appear in the final list, so that the facet-guessing method must fail.
We now combine this characterization of the success/failure of facet-guessing with our earlier structural properties of expander codes. Doing so yields the following result.

Corollary 1: For expander codes, the EFG algorithm will always succeed if there are $C_{1} \frac{\gamma_{c w}}{\gamma_{p c}}$ fractional pseudocodewords with likelihood higher than the ML codeword. Moreover, under this same condition, each iteration of RFG succeeds with constant probability

$$
p_{R F G} \geq \frac{\gamma_{c w}-C_{1} \gamma_{p c}}{2^{d_{c}-1}(1-R)+2} .
$$

Proof: From Lemma 4, the EFG algorithm fails if and only if every facet in $\left|A_{c}\right|$ also contains another fractional pseudocodeword with higher likelihood. But for expander codes, Lemma 3 yields that the size of the active set of any fractional pseudocodeword is upper bounded as

$$
\left|A_{p}\right| \leq n \gamma_{p c}
$$

while the size of active sets of any codeword is always $\left|A_{c}\right|=$ $n \gamma_{c w}$. Therefore, if there exist $C_{1}$ fractional pseudocodewords with likelihood higher than $c$, the total number of facets adjacent to these fractional pseudocodewords is at most $\gamma_{p c} C_{1} n$. Therefore, if we have

$$
\gamma_{p c} C_{1} n<n \gamma_{c w}
$$

it is impossible to completely cover $\mathrm{A}_{c}$ and EFG succeeds. Also RFG at each iteration selects a random facet and there are $\left(\gamma_{c w}-\right.$ $\left.\gamma_{p c} C_{1}\right) n$ facets that contain $c$ but not any fractional pseudocodeword with higher likelihood. The total number of facets is $|A|=$ $\left(2^{d_{c}-1}(1-R)+2\right) n$ and therefore each iteration of RFG has probability of success larger than

$$
\frac{\gamma_{c w}-C_{1} \gamma_{p c}}{2^{d_{c}-1}(1-R)+2}
$$

as claimed.

Notice that this corollary only provides a worst case bound. Indeed, the bound is achieved in a somewhat unlikely manner: it requires a set of fractional pseudocodewords all with higher likelihood than the ML codeword $\widehat{x}^{\mathrm{ML}}$, all of whose active sets are entirely contained within the active set $A\left(\widehat{x}^{\mathrm{ML}}\right)$ of the ML codeword, and all of whose active sets are pairwise disjoint. (See Fig. 1(b) for an illustration.) More typically, one could expect the facet guessing algorithm to work even if there are many more fractional pseudocodewords with higher likelihoods.

Our preliminary experimental results (Fig. 2) show that the facet guessing algorithm can indeed correct many pseudocodewords for which both sum-product and LP decoding fail. The experiments where performed by first running the sum-product decoder on two small codes. For the noise realizations where sum-product failed we used the Linear programming decoder (which almost always also failed). The random facet guessing algorithm was subsequently executed $N=20$ times. A list of 20 pseudocodewords was formed and the integral vertex with higher likelihood was the output. The RFG performance plot is made by counting all the noise realizations for which sum-product, LP decoding or the facet guessing steps where 


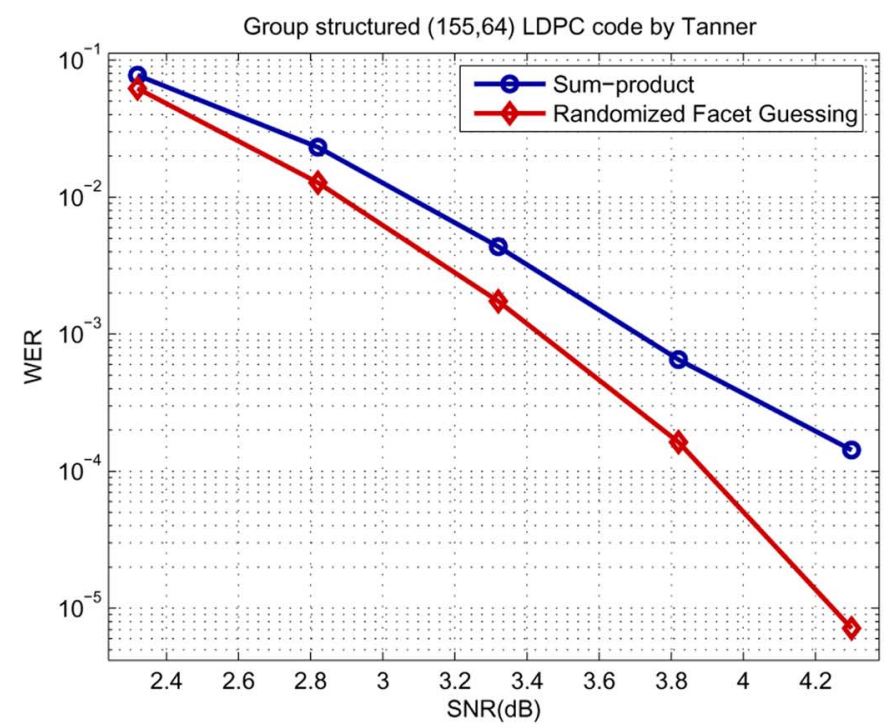

(a)

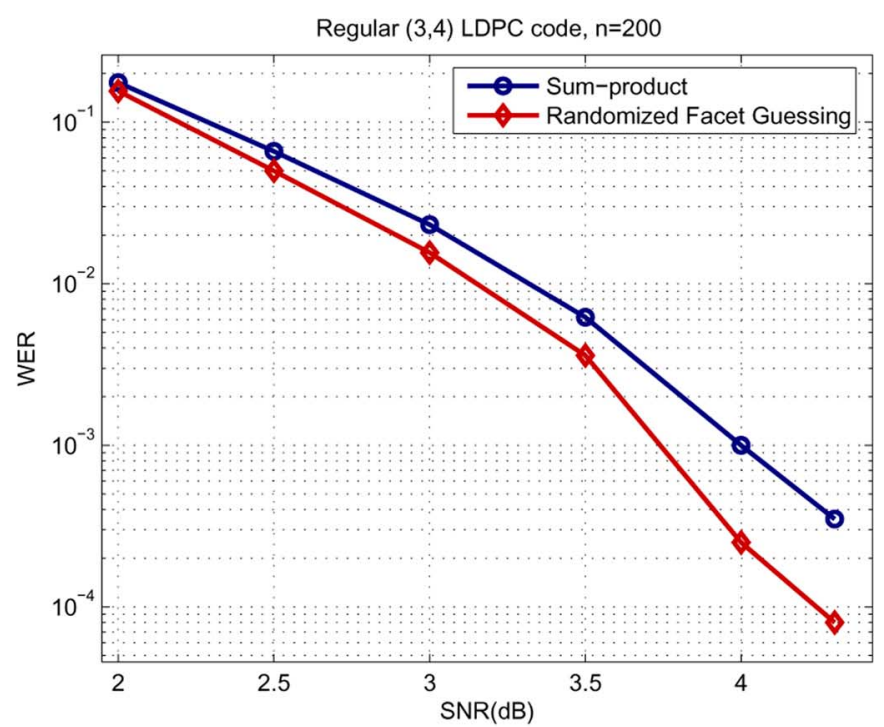

(b)

Fig. 2. Comparison of different decoding methods: standard sum-product decoding, and randomized facet-guessing (RFG) with $N=20$ iterations. The two panels show two different codes: (a) Tanner's group-structured code. (b) Random (3,4) LDPC code with $n=200$

successful. We observed that the RFG algorithm leads to a significant performance gains, frequently recovering the ML codeword in cases for which both sum-product and LP decoding fail. As shown in Fig. 2, the gains are pronounced for higher SNR, as high as $0.5 \mathrm{~dB}$ for the small blocklengths that we experimentally tested. The added complexity corresponds to solving a constant number of LP optimizations; moreover, the extra complexity is required only if LP decoding fails. Recently, Chertkov [34] proposed a facet guessing algorithm that uses critical loops to select appropriate facets and showed that the proposed scheme achieves essentially ML performance for the $[155,64,20]$ Tanner code.

It should be noted that while our theoretical result guarantees a correctable constant number of bad pseudocodewords, is unclear how these performance gains would scale in practice, for a fixed number of iterations, as the blocklength of the code grows. In other words, while there is a provable performance gain, for a constant number of guesses for expander graphs, this gain could be decreasing and asymptotically vanishing in the blocklength $n$. Investigating this performance gain relates to the interesting open questions that we discuss in Section V.

\section{IMPROVED THEORETICAL GUARANTEES}

The facet-guessing algorithm described in the previous section is based on choosing facets at random. Note that the chosen facet may either be of the forbidden set type, or the box constraint type (e.g., $0 \leq f_{i} \leq 1$ ). In this section, we describe a particular type of facet-guessing algorithm that chooses only box inequalities, and hence has the natural interpretation of a bit-guessing algorithm [18]. We show how this facet-guessing algorithm can be used to find the ML codeword in polynomial time, as long as the number of fractional pseudocodewords with higher likelihood is bounded by a polynomial.

\section{A. Random Bit Guessing}

The basic intuition underlying our random bit-guessing algorithm is simple. Since the LP decoding algorithm runs in polynomial time, we can afford to solve up to a polynomial number of linear programs to decode. Accordingly, we propose to choose a subset of bits, with size scaling as $c \log n$ for some constant $c>0$, and to try all $2^{c \log n}=n^{c}$ possible $0-1$ configurations indexed by bits in this subset. In one of these trials, the chosen configuration of $c \log n$ bits will match with the corresponding bits in the ML codeword. The algorithm will only fail if a "bad" pseudocodeword happens to coincide with the ML codeword in all $c \log n$ positions. The formal description of the algorithm is as follows:

\section{Randomized bit guessing (RBG) algorithm}

1) Run LP decoding: terminate if it outputs an integral codeword; otherwise go to step 2).

2) Choose $c \log n$ bits (randomly or deterministically), $x_{i_{1}}, x_{i_{2}}, x_{i_{3}}, \ldots, x_{i_{c \log n}}$.

3) Take as input the likelihood vector $\gamma$, and repeat the following loop for $2^{c \log n}=n^{c}$ times:

a) Consider a new 0-1 configuration out of the total $2^{c \log n}$ configurations for the $c \log n$ bits, say $\left(\alpha_{i_{1}}, \alpha_{i_{2}}, \alpha_{i_{3}}, \ldots, \alpha_{i_{c \log n}}\right)$.

b) Add the equations $x_{i_{j}}=\alpha_{i_{j}}$ for $j=1 \ldots c \log n$ to the set of inequalities defining the relaxed polytope and solve the linear program with objective vector $\gamma$ in $\mathcal{P}^{\prime}$. Save the optimal vertex $z_{i}$.

4) From the list of optimal LP solutions $\left\{z_{1}, \ldots, z_{c \log n}\right\}$, output the integral codeword with highest likelihood.

\section{B. Analysis}

Suppose that the set of $c \log n$ bits are chosen randomly. The main theorem of this section shows that this random 
bit-guessing (RBG) algorithm succeeds if there are at most polynomially many "bad" pseudocodewords. More formally, we let $M$ denote the number of pseudocodewords $x^{\mathrm{pc}}$ that

(a) have higher likelihood than the ML codeword $\widehat{x}^{\mathrm{ML}}$, and

(b) are adjacent to ML-codeword on the relaxed codeword polytope, meaning that the intersection $\mathrm{A}\left(\widehat{x}^{\mathrm{ML}}\right) \cap \mathrm{A}\left(x^{\mathrm{pc}}\right)$ is nonempty.

With this definition, we have the following.

Theorem 2: Given an $(\alpha, \delta)$-expander code with $\delta>\frac{1}{2}$, the RBG algorithm finds the ML-codeword with probability

$$
1-M /\left(n^{-c \log (1-\alpha)}\right) .
$$

Consequently, for any order $M=\mathcal{O}\left(n^{b}\right)$ of polynomial growth, the RBG algorithm succeeds with probability converging to one for all

$$
c>b / \log (1-\alpha)
$$

Proof: By the code symmetry of the relaxed polytope [5], we may assume without loss of generality that $\widehat{x}^{\mathrm{ML}}$ is the all-zeroes codeword (although the algorithm does not know this information). If $\widehat{x}^{\mathrm{ML}}$ is the all-zeroes word, then the key iteration of the RBG algorithm is the step at which it sets $x_{i_{j}}=0$ for $j=1 \ldots c \log n$. From Proposition 1, since the graph is an $(\alpha, \delta)$-expander code, every pseudocodeword has at least $\alpha n$ fractional coordinates. Therefore, a randomly chosen bit from any pseudocodeword will be integral with probability at most $1-\alpha$. Consequently, if we force a set $c \log n$ bits to zero (as in the key step described above), then the probability that all the bits fall outside the fractional support of any given pseudocodeword is at most $(1-\alpha)^{c \log n}$. Otherwise stated, with probability at least $1-(1-\alpha)^{c \log n}$, a random selection of $c \log n$ bits will exclude any particular pseudocodeword as a possible output of the RBG algorithm. By a union bound, any set of $M$ pseudocodewords are excluded with probability at least

$$
1-M(1-\alpha)^{c \log n}=1-M n^{c \log (1-\alpha)} .
$$

Consequently, if there are at most $M$ pseudocodewords with likelihood higher than the ML codeword, then the RBG algorithm will succeed with at least this probability.

In order to complete the proof, we need to show that it is sufficient to exclude only higher likelihood pseudocodewords that are also adjacent on the relaxed polytope $\mathcal{P}$ to the all-zeroes ML codeword. In order for the all-zeros $\widehat{x}^{\mathrm{ML}}$ to not be the output of the restricted LP at the key step (in which the set of $c \log n$ bits are set to zero), there must exist a pseudocodeword in the restricted polytope

$$
\mathcal{P} \cap\left(\cap_{j=1}^{c \log n}\left\{x_{i_{j}}=0\right\}\right)
$$

with higher likelihood. Any such pseudocodeword is certainly adjacent to the all-zeroes codeword, since they share all the box constraints $x_{i_{j}}=0$. Therefore, it is sufficient to exclude only "bad" pseudocodewords that are adjacent to the ML-codeword on the relaxed polytope.

\section{CONCLUSION}

In this paper, we have investigated the structure of the polytope that underlies both the LP method and the sum-product algorithm for decoding of low-density parity check codes. For codes based on suitable expander graphs, we proved a number of structural properties of this polytope, including the fact that any (fractional) pseudocodeword has at least a constant fraction of nonintegral bits, and that the number of active sets differ substantially between pseudocodewords and codewords. Inspired by these structural properties, we proposed a number of efficient decoding algorithms that offer quantifiable improvements over basic LP decoding. First, we described a facet-guessing algorithm and provided both theoretical and empirical results on the performance gains that it achieves. We also proposed a randomized bit-guessing algorithm, and proved that it can still recover the ML codeword as long as there are at most a polynomial number of pseudocodewords with higher likelihood.

The results of this paper raise an interesting question concerning the structure of pseudocodewords in various code families. Previous work by Koetter and Vontobel [10] established that for any bit-check regular LDPC code, there exist pseudocodewords for the additive white Gaussian noise (AWGN) channel with sublinear pseudoweight. This fact implies that standard LP decoding cannot have an error exponent for the AWGN, meaning an exponential decay in error probability. ${ }^{2}$ While standard LP decoding can be compromised by a single "bad" pseudocodeword, the improved decoding procedures in this paper can still tolerate a polynomial number of such bad pseudocodewords. Therefore, it would be interesting to identify families of codes which have a bounded number of such bad pseudocodewords. Theoretical understanding of properly guided facet guessing techniques, such as the one developed by Chertkov [34], and connections to bit guessing and decimation schemes in message-passing [23]-[25] are also interesting directions for future work.

\section{ACKNOWLEDGMENT}

The authors would also like to thank the anonymous reviewers for their constructive suggestions that helped to improve the paper.

\section{REFERENCES}

[1] R. G. Gallager, Low-Density Parity Check Codes. Cambridge, MA: MIT Press, 1963.

[2] T. Richardson and R. Urbanke, "The capacity of low-density parity check codes under message-passing decoding," IEEE Trans. Inf. Theory, vol. 47, pp. 599-618, Feb. 2001.

[3] A. Ashihkmin, G. Kramer, and S. ten Brink, "Extrinsic information transfer functions: Model and erasure channel properties," IEEE Trans. Inf. Theory, vol. 50, pp. 2657-2673, 2004.

[4] T. Richardson and R. Urbanke, Modern Coding Theory. Cambridge, U.K.: Cambridge Univ. Press, 2008.

[5] J. Feldman, M. J. Wainwright, and D. R. Karger, "Using linear programming to decode binary linear codes," IEEE Trans. Inf. Theory, vol. 51, pp. 954-972, Mar. 2005.

[6] J. Feldman, D. R. Karger, and M. J. Wainwright, "Using linear programming to decode LDPC codes," in Conf. Inf. Sci. Syst., Mar. 2003.

${ }^{2}$ Subsequent work [14] showed that LP decoding does have an error exponent if the log likelihoods are suitably thresholded; however, doing so discards potentially useful information. 
[7] J. Feldman, D. R. Karger, and M. J. Wainwright, "Linear programming-based decoding of turbo-like codes and its relation to iterative approaches," in Proc. 40th Ann. Allerton Conf. Commun., Control, Comput., Oct. 2002.

[8] M. J. Wainwright, T. S. Jaakkola, and A. S. Willsky, "MAP estimation via agreement on (hyper)trees: Message-passing and linear programming approaches," in Proc. 40th Allerton Conf. Commun., Control Comput., Oct. 2002.

[9] M. J. Wainwright, T. S. Jaakkola, and A. S. Willsky, "Exact MAP estimates via agreement on (hyper)trees: Linear programming and message-passing," IEEE Trans. Inf. Theory, vol. 51, pp. 3697-3717, Nov. 2005.

[10] R. Koetter and P. O. Vontobel, "Graph-covers and iterative decoding of finite length codes," in Proc. 3rd Int. Symp. Turbo Codes, Sep. 2003.

[11] C. Daskalakis, A. G. Dimakis, R. M. Karp, and M. J. Wainwright, "Probabilistic analysis of linear programming decoding," in Proc. 18th Ann. Symp. Discrete Algorithms (SODA), Jan. 2007.

[12] J. Feldman, T. Malkin, R. A. Servedio, C. Stein, and M. J. Wainwright, "LP decoding corrects a constant fraction of errors," IEEE Trans. Inf. Theory, vol. 53, no. 1, pp. 82-89, Jan. 2007.

[13] P. Vontobel and R. Koetter, "Lower bounds on the minimum pseudoweight of linear codes," in Int. Symp. Inf. Theory (ISIT '04), Chicago, IL, Jun. 2004

[14] J. Feldman, R. Koetter, and P. O. Vontobel, "The benefit of thresholding in LP decoding of LDPC codes," in Proc. Int. Symp. Inf. Theory, 2005, pp. 307-311.

[15] P. O. Vontobel and R. Koetter, "Towards low-complexity linear-programming decoding," in Proc. Int. Conf. Turbo Codes and Related Topics, Munich, Germany, Apr. 2006.

[16] M. H. Taghavi and P. H. Siegel, "Adaptive linear programming decoding," in Proc. IEEE Int. Symp. Inf. Theory, Seattle, WA, Jul. 2006.

[17] M. P. C. Fossorier, "Iterative reliability-based decoding of low-density parity check codes," IEEE Trans. Inf. Theory, pp. 908-917, May 2001.

[18] H. Pishro-Nik and F. Fekri, "On decoding of LDPC codes over the erasure channel," IEEE Trans. Inf. Theory, vol. 50, pp. 439-454, 2004.

[19] K. Yang, J. Feldman, and X. Wang, "Nonlinear programming approaches to decoding low-density parity-check codes," IEEE J. Sel. Areas Commun., vol. 24, pp. 1603-1613, Aug. 2006.

[20] S. Draper, J. Yedidia, and Y. Wang, "ML decoding via mixed-integer adaptive linear programming," in Proc. IEEE Int. Symp. Inf. Theory (ISIT), 2007.

[21] M. Chertkov and V. Y. Chernyak, "Loop calculus helps to improve belief propagation and linear programming decoding of LDPC codes," in Proc. Allerton Conf. Commun., Control Computing, Monticello, IL, Sep. 2006.

[22] D. Bertsimas and J. Tsitsikilis, Introduction to Linear Optimization. Belmont, MA: Athena Scientific, 1997.

[23] M. J. Wainwright and E. Maneva, "Lossy source coding by message-passing and decimation over generalized codewords of LDGM codes," in Proc. Int. Symp. Inf. Theory, Adelaide, Australia, Sep. 2005, arxiv:cs.IT/0508068.

[24] M. Mézard, G. Parisi, and R. Zecchina, "Analytic and algorithmic solution of random satisfiability problems," Science, vol. 297, pp. 812-812, 2002.

[25] A. Montanari, F. Ricci-Tersenghi, and G. Semerjian, "Solving constraint satisfaction problems through belief propagation-guided decimation," in Proc. Allerton Conf., 2007.

[26] R. M. Tanner, "A recursive approach to low complexity codes," IEEE Trans. Inf. Theory, vol. IT-27, pp. 533-547, Sep. 1980.
[27] F. Kschischang, B. Frey, and H.-A. Loeliger, "Factor graphs and the sum-product algorithm," IEEE Trans. Inf. Theory, vol. 47, pp. 498-519, Feb. 2001.

[28] E. Berlekamp, R. McEliece, and H. van Tilborg, "On the inherent intractability of certain coding problems," IEEE Trans. Inf. Theory, pp. 384-386, 1978

[29] K. Yang, X. Wang, and J. Feldman, "Cascaded formulation of the fundamental polytope of general linear block codes," in Proc. IEEE Int. Symp. Inf. Theory (ISIT), 2007.

[30] M. J. Wainwright and M. I. Jordan, Graphical Models, Exponential Families, and Variational Inference Univ. Calif., Dep. Statist., Berkeley, Tech. Rep., No. 649, 2003.

[31] M. Sipser and D. Spielman, "Expander codes," IEEE Trans. Inf Theory, vol. 42, pp. 1710-1722, Nov. 1996.

[32] A. Barg and G. Zémor, "Error exponents of expander codes," IEEE Trans. Inf. Theory, vol. 48, pp. 1725-1729, 2002.

[33] G. M. Ziegler, Lectures on Polytopes. New York: Springer-Verlag, 1995.

[34] M. Chertkov, "Reducing the error floor," in Proc. IEEE Inf. Theory Workshop (ITW), 2007.

Alexandros G. Dimakis (S'05) received the Ph.D. degree in 2008 from the University of California, Berkeley (UC Berkeley). He received the Diploma degree in electrical and computer engineering from National Technical University of Athens, Greece, in 2003.

He was a Postdoctoral Scholar at the Center for the Mathematics of Information at the California Institute of Technology (Caltech), Pasadena, and he is currently an Assistant Professor at the Electrical Engineering Department, University of Southern California, Los Angeles. His research interests include communications, coding theory, signal processing, and networking, with a current focus on network coding, message passing algorithms and sparse graph codes.

Dr. Dimakis received the Eli Jury dissertation award in 2008, two outstanding paper awards, a UC Berkeley Regents Fellowship and a Microsoft Research Fellowship.

Amin A. Gohari (S'06) is currently working towards the $\mathrm{Ph} . \mathrm{D}$. degree with the Electrical Engineering Department, University of California. He received the B.Sc. degree from Sharif University of Technology and joined Berkeley in 2004.

His research interests include coding and multiuser information theory.

Martin J. Wainwright (M'05) received the Ph.D. degree in electrical engineering and computer science (EECS) from Massachusetts Institute of Technology (MIT), Cambridge.

$\mathrm{He}$ is currently an Assistant Professor with the University of California, Berkeley, with a joint appointment between the Department of Statistics and the Department of Electrical Engineering and Computer Sciences. His research interests include statistical signal processing, coding and information theory, statistical machine learning, and high-dimensional statistics.

Dr. Wainwright has been awarded an Alfred P. Sloan Foundation Fellowship, an NSF CAREER Award, the George M. Sprowls Prize for his dissertation research, a Natural Sciences and Engineering Research Council of Canada 1967 Fellowship, and several outstanding conference paper awards. 